\title{
New aspects of the structure and mode of action of the human cathelicidin LL- 37 revealed by the intrinsic probe p-cyanophenylalanine
}

\author{
Daniela Xhindoli ${ }^{*}$, Francesca Morgera ${ }^{*}$, Ursula Zinth ${ }^{\mathrm{I}}$, Roberto Rizzo ${ }^{*}$, Sabrina Pacor ${ }^{*}$, Alessandro \\ Tossi $^{*}$ \\ *Department of Life Sciences, University of Trieste, via Giorgeri 5, 34127 Trieste, Italy \\ ${ }^{\mathbb{I}}$ Department of Chemistry, Technical University of Munich, D-85747 Garching, Germany
}

\begin{abstract}
The human cathelicidin peptide LL-37 is an important effector of our innate immune system and contributes to host defence with direct antimicrobial activity, immunomodulatory properties and by stimulating wound healing. Its sequence has evolved to confer specific structural characteristics that strongly affect these biological activities, and that differentiates it from orthologues of other primate species. Here we report a detailed study of the folding and self-assembly of this peptide in comparison to rhesus monkey peptide RL-37, taking into account the different stages of its trajectory from bulk solution to contact with, and insertion into, biological membranes. Phe residues in different positions throughout the native sequences of LL-37 and RL-37 were systematically replaced with the non-invasive fluorescent and IR probe p-cyanophenylalanine. Steady-state and time-resolved fluorescence studies showed that LL-37, in contrast to RL-37, forms oligomers with a loose hydrophobic core in physiological solutions, which persist in the presence of biological membranes. FTIR and surface plasmon resonance studies also indicated different modes of interaction for LL-37 and RL-37 with anionic and neutral membranes. This correlated with a distinctly different mode of bacterial membrane permeabilization, as determined using a flow cytometric method involving impermeant fluorescent dyes linked to polymers of defined sizes.
\end{abstract}

Short title: Structural and functional characterization of the antimicrobial peptide LL-37

Key words: LL-37, RL-37, oligomerization, p-cyano-L-phenylalanine, time-resolved fluorescence, Surface Plasmon Resonance

\begin{abstract}
Abbreviations: AFM, atomic force microscopy; AMP, antimicrobial peptide; ATR, attenuated total reflection, DPPC, 1,2-dipalmitoyl-sn-glycero-3-phosphocholine, DPPG, 1,2-dipalmitoyl-snglycero-3-phospho-rac-(3-lysyl(1-glycerol)); $\mathrm{F}_{\mathrm{CN}}$, p-cyano-L-phenylalanine; FITC-Dx, Flourescein isothiocyanate-dextran; FTIR, Fourier transform infrared; $\mathrm{K}_{\mathrm{D}}$, dissociation constant; LUVs, large unilamellar vesicles; LUV ${ }^{(-)}$, anionic LUVs; LUV ${ }^{(0)}$, neutral LUVs; PC, L- $\alpha$-phosphatidylcholine; PG, L- $\alpha$-phosphatidylglycerol; PI, propidium iodide; SM, sphingomyelin, SPR, surface plasmon resonance; SPB, sodium phosphate buffer, TFE, trifluoroethanol.
\end{abstract}




\section{Introduction}

LL-37, the only human cathelicidin, is an essential component of our innate immune system and plays diverse roles in protection against microbial infection. It is capable of directly inactivating bacteria, at micro molar concentrations, due to its capacity to interact with various components of bacterial membranes (LPS, lipoteichoic acid, peptidoglycan and phospholipids) and then halting bacterial growth by interfering with cell wall synthesis [1,2] or by disrupting membrane integrity [3] and likely membrane-bound protein machinery. In addition, LL-37 has a potent anti-endotoxin activity, binding efficiently to and neutralizing LPS and lipoteichoic acid, thus reducing inflammation [4,5]. It further modulates inflammatory responses by interfering with toll-like receptor signaling and inhibiting pro-inflammatory cytokine release from human monocytes [6]. LL-37 also acts as a signal molecule, helping to recruit circulating immune cells to sites of infection by interacting with different types of cellular receptors [7]. Lastly, it appears to play a key role in repair of damaged tissue and wound closure by promoting neovascularization and reepithelialization of healing skin [8,9] and by stimulating fibroblast proliferation [10].

LL-37 is a peptide of just 37 residues, with charge +6 under physiological conditions. It switches from a disordered conformation in aqueous solution, to an amphipathic helix spanning residues 231 of membrane-like environments, but also in the presence of appropriate salt ions $[11,12]$. While it is unusual for AMPs to adopt a helical structure in the absence of membranes, in LL-37 the acidic and basic residues are arranged so as to form a net of intra- and inter-molecular salt-bridges. These strongly stabilize this helical conformation and drive LL-37 self-association into oligomeric bundles. In this way, it can also partly shield the hydrophobic surface of the amphipathic helix from the aqueous environment. Oligomerization significantly affects LL-37 biological activities and appears to favor its capacity to stimulate host cell proliferation $[3,10,11,13]$. On the other hand the propensity to oligomerize also makes this peptide prone to sequestration by medium or serum components, or by the components of the bacterial outer wall layers, affecting its bactericidal capacity [13]. Despite a 70\% homology in primary sequence, the rhesus orthologue RL-37 does not fold or oligomerize in aqueous salt solutions, due to a less favorable salt-bridging arrangement. This results in a more efficient and less environment-sensitive antimicrobial activity of RL-37, especially against Gram-positive bacteria, likely due to an increased capacity to reach and disrupt bacterial membranes [13,14]. Conversely, RL-37 has a reduced capacity to affect host cells, which may correlate with a reduced capacity to interact with their membranes $[13,14,15]$.

The different behavior of the two peptides in bulk solution thus strongly affects the manner in which they interact with and alter bacterial or host cells. Using flow cytometry and atomic force microscopy, we have previously observed that the oligomeric human peptide appears to form discreet channels in anionic membranes, compatible with toroidal pores, whereas the monomeric RL-37 results in the rapid formation of smaller, irregular lesions [3,15]. Apart from pore formation, it has been proposed that helical AMPs inactivate bacteria by also disrupting the coordinated and highly dynamic functioning of essential, membrane bound protein machineries [16], and it is plausible that the two peptides differ in these activities too. In order to better understand how the oligomeric form of the peptide in solution drives membrane effects, we have systematically replaced the phenylalanine residues in the native sequence of LL-37 and RL-37 with the isosteric unnatural amino acid p-cyanophenylalanine $\left(\mathrm{Phe}_{\mathrm{CN}}\right.$ or $\left.\mathrm{F}_{\mathrm{CN}}\right)$. This fluorophore is a promising probe for conformational studies given its sensitivity to the local environment.

Phe $_{\mathrm{CN}}$ is quite non-invasive and has an emission quantum yield five times greater than that of Phe [17]. The $\mathrm{CN}$ moiety has a polarity intermediate between an amide and a methyl group, allowing this side-chain to place itself either near the polar surface of a protein or in its hydrophobic interior, without significantly perturbing peptide's secondary structure or physicochemical properties. $\mathrm{F}_{\mathrm{CN}}$ 
has been used to replace either Phe or Tyr residues in studies of protein folding [18,19], formation of amyloid fibrils [20], and protein-protein and protein-membrane interactions [17,21]. Its emission is dynamically quenched by halogen ions [22] and in contrast to most fluorophores, is reduced in apolar environments [17]. Recent detailed studies of its photophysical properties in different solvents have found that the fluorescence quantum yield and decay are both highly sensitive to hydrogen bonding interactions involving the $\mathrm{CN}$ group [23,24,25], which makes it a very useful probe for the hydration/dehydration status of a protein, and explains why its fluorescence decreases in aprotic solvents. $\mathrm{F}_{\mathrm{CN}}$ is also a useful IR probe, as the $\mathrm{C} \equiv \mathrm{N}$ stretching band is in a transparent region of the typical protein IR spectrum, and is strongly blue-shifted when the side-chain moves from a polar to an apolar environment $[17,26]$.

We have prepared three fluorescent analogs of LL-37 and two of RL-37 and report here on both their steady state and time-resolved fluorescence under several different conditions, in order to follow in detail how helical formation occurs in different regions of the peptides as they change environment. We then compared these results with those from FTIR and surface plasmon resonance studies of membrane interactions and correlated them with flow cytometric results on the mode of bacterial membrane permeabilization. Apart from providing new insights into the behavior of an important human innate immune effector, these studies indicate new aspects of $\mathrm{Phe}_{\mathrm{CN}}$ as a powerful intrinsic probe for studies on polypeptide conformation and interactions.

\section{Material and methods}

\subsection{Peptide synthesis and purification}

Solid-phase synthesis of LL-37, RL-37 and p-cyanophenylalanine (PheCN) analogues were performed on an automated, microwave assisted Liberty peptide synthesizer (CEM Corporation, Matthews, NC) using FmocSer-Pal-PEG resin (substitution $0.24 \mathrm{mmol} / \mathrm{g}$ ). Single Phe residue at each position was replaced by $\mathrm{Phe}_{\mathrm{CN}}$, using Fmoc-Phe ${ }_{\mathrm{CN}}-\mathrm{OH}$ for the coupling reaction (Sigma Aldrich). All other protected amino acids were from Novabiochem (Merck Millipore). The two reference peptides were synthesized as C-terminal amides using a PAL-PEG-PS resin (Applied Biosystems). Couplings were generally carried out with a fourfold excess of incoming amino acids in N-methyl pyrrolidone (NMP) and were activated with either PyBOP or HCTU (Sigma). Cleavage of the completed peptides from the resin was effected using a version of the reagent $\mathrm{K}$ (TFA $87 \%$, TPIS $2 \%, \mathrm{H}_{2} \mathrm{O} 3 \%$ and DODT $8 \%$ ), treating for 4 hours at room temperature, under continuous stirring. Crude peptides were precipitated in cold methyl tert-butyl ether (TBME), washed two times with Diethyil ether, and dried under a nitrogen flux.

Crude peptides were purified on a preparative Phenomenex Jupiter Proteo C18 column $(21$ x $250 \mathrm{~mm}, 10 \mu \mathrm{m}$, $90 \AA$ Å) using a 30-60\% acetonitrile in 60 min. Peptide purity was confirmed by ESI-MS on an Esquire 4000 mass spectrometer (Bruker Daltonics, Billerica MA). Peptides were then repeatedly lyophilized from 10mM $\mathrm{HCl}$ to remove TFA, before storage in the dark at $-20^{\circ} \mathrm{C}$. Peptide concentrations of stock solutions were determined optically using the relatively strong molar extinction coefficient of Phe $\mathrm{CN}_{\mathrm{CN}}, \varepsilon_{240}=13000 \mathrm{M}^{-1} \mathrm{~cm}^{-1}$ [17], another advantage of using this residue.

\subsection{Liposome preparation}

Anionic LUVs (large unilamellar vesicles, $\mathrm{LUV}^{(-)}$) for spectroscopic studies were prepared with the anionic phospholipids L- $\alpha$-phosphatidylglycerol (PG, egg yolk) and cardiolipin (dPG, bovine heart) at 95:5 w/w. Neutral LUVs $\left(\mathrm{LUV}^{(0)}\right)$ were prepared with zwitterionic lipids L- $\alpha$-phosphatidylcholine (PC, egg yolk), sphingomyelin (SM, egg yolk), together with cholesterol (Ch, ovine wool) (40:40:20, w/w). For surface plasmon resonance studies, $\mathrm{LUV}^{(-)}$were prepared with 1,2-dipalmitoyl-sn-glycero-3-phosphocholine (DPPC) and 1,2-dipalmitoyl-sn-glycero-3-[phospho-rac-(3-lysyl(1-glycerol)) (DPPG) at 4:1 w/w and LUV ${ }^{(0)}$ with DPPC, as these allowed optimal coverage of the L1 chip. All lipids were obtained from Avanti Polar Lipids. 
Lipids were first dissolved and mixed in Chloroform/Methanol (2:1). The organic solvent was then removed under a stream of argon and vacuum-dried overnight. The lipid cake was re-suspended to a concentration of $4 \mathrm{mg} \mathrm{lipid} / \mathrm{ml}$ in $100 \mathrm{mM}$ sodium phosphate buffer (SPB) by spinning the flask at $45^{\circ} \mathrm{C}$ (above the transition temperature of the lipids). The resulting multilammellar vesicle suspensions were then disrupted by several freeze-thaw cycles prior to extrusion through successive polycarbonate filters with $1 \mu \mathrm{m}, 0.4 \mu \mathrm{m}$ and $0.1 \mu \mathrm{m}$ pores, using a Mini-Extruder (Avanti Polar Lipids, Inc.). LUVs were conserved at $4^{\circ} \mathrm{C}$ and always used within one or two days of preparation.

\subsection{Circular dichroism}

CD spectroscopy was performed on a J-715 spectropolarimeter (Jasco, Tokyo, Japan) using $2 \mathrm{~mm}$ path length quartz cells and $20 \mu \mathrm{M}$ peptides (referred to peptide chains, not molecules) in $\mathrm{H}_{2} \mathrm{O}, 10 \mathrm{mM}$ sodium phosphate buffer (SPB), $139 \mathrm{mM} \mathrm{NaCl}$ in sodium phosphate buffer (PBS), 50\% TFE and in presence of large unilamellar vesicles. (PG/dPG 95:5) and (PC/SM/Ch 40:40:20) liposomes were used as models for anionic bacterial and neutral host membranes, respectively. The helical content (\% helix) was estimated from the molar ellipticity at $222 \mathrm{~nm}$ [27]. All displayed CD spectra are the accumulation of at least three scans.

\subsection{Steady-state fluorescence}

Fluorescence spectra of cyanophenylalanine-containing peptides were obtained using either a Perkin Elmer LS50B instrument or a Fluorolog, Jabin Yvon Horiba, and $5 \mu \mathrm{M}$ peptide solutions in different environments: i) pure $\mathrm{H}_{2} \mathrm{O}$ ii) $10 \mathrm{mM}$ or $100 \mathrm{mM}$ sodium phosphate buffer (SPB), iii) titrating between $0-200 \mathrm{mM} \mathrm{NaCl}$ in $\mathrm{H}_{2} \mathrm{O}$ iv) anionic or neutral LUVs in $100 \mathrm{mM} \mathrm{SPB}$. Excitation of $\mathrm{Phe}_{\mathrm{CN}}$ was at $240 \mathrm{~nm}$ and emission was monitored at $300 \mathrm{~nm}$ at $22.5^{\circ} \mathrm{C}$, using slits of $2 \mathrm{~nm}$.

\subsection{Time Resolved fluorescence}

Fluorescence decays were determined at $22.5^{\circ} \mathrm{C}$ using a FluoTime200 Time-resolved spectrometer (PicoQuant, Berlin, Germany) with a PDL 800-D picosecond pulsed diode laser driver unit, a PicoHarp timecorrelated single photon counting system and PMA-182 photo multiplier tubes (based on H5783 photosensor modules, Hamamatsu). Excitation pulses at $247 \mathrm{~nm}$ were LED generated with a pulse width of 840 ps. The repetition frequency of the excitation pulses was set to $10 \mathrm{MHz}$. The setup was equipped with polarizes and a cuvette holder (TLC 50, Quantum northwest, WA, USA) with Peltier temperature regulation and magnetic stirrer. The fluorescence decays were measured under the same conditions as steady state determinations. The obtained data were subject to either mono- or bi-exponential fit using FluoFit (PicoQuant) and taking into account the non-uniform instrumental response function through reconvolution. IRFs were determined by recording the scattering of dilute solutions of Ludox (Sigma Aldrich) in water.

\subsection{FTIR}

Transmission and ATR-FTIR spectra were collected as described previously [28], using Vertex 70 Bruker FTIR spectrometer equipped with a Mid Band Mercury-Cadmium-Telluride detector (MCT D316) and a HORIZON multiple reflectance ATR accessory (Harrick Scientific Products, Inc.). Transmission spectra (512 scans, spectral resolution $2 \mathrm{~cm}^{-1}$ ) were collected using $2 \mathrm{mM}$ peptide solutions in $\mathrm{D}_{2} \mathrm{O}$, placed between $\mathrm{CaF}_{2}$ windows spaced 25 microns apart in a dismountable liquid cell (Harrick Scientific Products, Inc.). ATR spectra (128 scans with a resolution of $\left.4 \mathrm{~cm}^{-1}\right)$ using $20 \mu \mathrm{M}$ peptide incubated with phospholipid vesicles $(0.4$ $\mathrm{mM}$ phospholipids) in $5 \mathrm{mM} \mathrm{SPB}$ at $\mathrm{pH} 7.0$ that were then deposited on a trapezoidal $45^{\circ}$ Germanium ATR crystal $(50 \mathrm{~mm} \times 10 \mathrm{~mm} \times 2 \mathrm{~mm})$ at $37^{\circ} \mathrm{C}$. The buffer evaporation resulted in lipid multi-layers on which the spectrum was recorded [29]. The background was collected directly on a clean internal reflection element (IRE).

\subsection{Surface Plasmon Resonance}

Both Inter-peptide interactions and peptide-membrane interactions were studied using an X100 instrument (Biacore, GE Lifesciences). In the first case, LL-37 (58 $\mu \mathrm{g} / \mathrm{ml})$ in sodium acetate buffer ( $\mathrm{pH} 5)$ was anchored to a CM5 chip, following the protocol provided with the Biacore EDC/NHS coupling kit. The level of the 
substitution reached after immobilization and regeneration was equivalent to $50 \mathrm{RU}$, so that the molecules should be well separated on the surface $(\sim 16 \mathrm{~nm})$, given that $100 \mathrm{RU}$ corresponds to $0.1 \mathrm{ng} / \mathrm{mm}^{2}$ of the surface [30]. Peptide-peptide interaction studies were performed either in mildly basic conditions using 10 fold diluted HBS-EP ( $\mathrm{pH} 7.4$, provided by GE Lifesciences), or in acidic conditions using sodium acetate $10 \mathrm{mM} / 1 \mathrm{mM}$ EDTA (pH 5). Free LL-37 in solutions from 4 to $64 \mu \mathrm{M}$ was flowed in successive cycles for 3 min across the surface of the LL-37-modified chip to collect sensorgrams, and the analyte was then washed away after each cycle by injecting $50 \mathrm{mM} \mathrm{NaOH}$ for 60 seconds.

For peptide-membrane interactions, an L1 sensor chip with dextran matrix modified with lipophilic chains was used to captured anionic or neutral liposomes [31]. Anionic LUVs were composed of: dipalmitoylphosphatidylcholine and 1.2-dipalmitoylphosphatidylglycerol (DPPC/DPPG, 4:1) and neutral LUVs of only DPPC. Liposomes in $139 \mathrm{mM}$ PBS at $1 \mathrm{mM}$ concentration were flowed at $5 \mu \mathrm{l} / \mathrm{min}$ over the surface till a plateau was reached (7000-8000 RU) and $1 \mathrm{mM}$ EDTA in PBS buffer was then flowed overnight to ensure a stable the bilayer. Bovine serum albumin (BSA), at $0.1 \mathrm{mg} / \mathrm{ml}$ in PBS, was used as a negative control to confirm complete coverage of surface with LUVs as described [32]. Peptide solutions $(0.25-16 \mu \mathrm{M})$ in PBS $150 \mathrm{mM}$, EDTA $1 \mathrm{mM})$ were flowed over the membrane layer for 240 seconds, followed by a dissociation time of $1800 \mathrm{sec}$. For RL-37, where the system reached a steady state during injection of the sample, it was possible to measure an affinity constant using suitable models for peptide-membrane interaction, and the numerical integration analysis provided by the BIACORE X100 Evaluation Software.

\subsection{Antibacterial activity and membrane permeabilization}

The effect of peptides on bacterial growth kinetics were determined on mid-logarithmic E. coli $M L-35\left(10^{6}\right.$ cells $/ \mathrm{ml}$ in 5\% tryptic soy broth in SPB) as described previously [13]. Flow cytometric analyses of membrane permeabilization were performed on a mid-exponential culture of $S$. aureus ATCC25923 (10 cell $/ \mathrm{ml}$ ) in $20 \% \mathrm{MH}$ broth in $10 \mathrm{mM} \mathrm{SPB}$, treated with 2, 4 and $8 \mu \mathrm{M}$ peptide concentrations for 60 min at $22^{\circ}$ C. PI (propidium iodide, Sigma-Aldrich) and FD-4, -10, and -40 (FITC-labelled dextran 4-40 kDa, Sigma-Aldrich) were then added to final concentrations of $10 \mu \mathrm{g} / \mathrm{ml}$ and $0.25 \mathrm{mg} / \mathrm{ml}$ respectively. Cells were analyzed with in an FC500 flow cytometer (Beckman Coulter). For each measurement, 10000 events were collected, stored as list mode files, and analyzed using FCS Express (DeNovo Software).

\section{Results and Discussions}

\section{1) Use of steady-state and time-resolved fluorescence to follow the conformational equilibrium of LL-37under different conditions}

Unlike most helical AMPs that are not structured in an aqueous environment, LL-37 has a tendency to adopt a helical structure at physiological salt concentrations and $\mathrm{pH}$, driving its selfassembly into oligomers. Our recent studies based on replacing Phe $\mathrm{P}^{5}$ in the sequence of LL-37 with the photochemically active cross-linking residue benzoyl-phenylalanine (Bpa) showed that LL-37 is in equilibrium between monomeric and oligomeric forms up to hexamers, in PIL buffer that mimics the ion composition of plasma [3]. Furthermore LL-37 only partly dissociates to a lower order of oligomerization when in contact with bacterial- and host-like model membranes. This behaviour is distinctly different from that of the rhesus macaque orthologue, RL-37, which is not structured in aqueous solution and adopts a helical conformation only in the presence of bacterial membranes. Given the previously observed relevance of self-association to biological activities, we endeavoured to better define the characteristics of the native monomers and oligomers, both in bulk solution and at the surface of biological membranes.

To probe the inter- and intramolecular environment in different regions of LL-37 and RL-37, we have exploited the presence of Phe residues scattered throughout the sequence, and systematically effected single substitutions with $\mathrm{F}_{\mathrm{CN}}$ in available positions 5, 17 or 27 in LL-37 and 5 or 27 in RL37 (see Table 1). In Figure 1, the spatial arrangement of these residues is shown in the context of 
the published helical NMR structure of LL-37 obtained in the presence of D8PG micelles [11]. Their relative positioning on dimerization (considering either a parallel or antiparallel chain arrangement) is also schematically shown. The replacement of Phe by $\mathrm{Phe}_{\mathrm{CN}}$ was quite noninvasive, both structurally, judging from the CD spectra of the peptides in different environments (see supplementary Figure S1) and functionally, as the capacity of the peptides to inhibit the growth of E. coli (see supplementary Figure S2) was comparable to that of the parent peptide LL37 [13].

Two reference peptides $\left(\mathrm{GF}_{\mathrm{CN}} \mathrm{KG}\right.$ and $\left.\mathrm{GF}_{\mathrm{CN}} \mathrm{FKG}\right)$ were also prepared. The former was designed to have the fluorophore fully exposed to the aqueous environment, next to a charged residue to ensure full solvation, and is representative of $\mathrm{F}^{17}$ in LL-37 or $\mathrm{F}^{27}$ in either LL-37 or RL-37 (see Table 1). $\mathrm{GF}_{\mathrm{CN}} \mathrm{FKG}$ is instead representative of the $\mathrm{F}^{5}$ environment in both peptides, where the presence of the flanking $\mathrm{F}^{6}$ residue might affect the emission characteristics of the fluorophore. Furthermore, these two reference peptides should neither structure/assemble nor interact with the surface of the membranes.

$\mathrm{F}_{\mathrm{CN}}$ has several advantages as an internal fluorescent probe, as its emission intensity has a strong positive correlation with the Kamlett-Taft solvatochromic parameter $\alpha$ of the solvent (hydrogen bond donor capacity). Unlike most other fluorescent probes, the quantum yield thus increases in polar solvents such as water, due to their H-bonding capacity, with respect to aprotic solvents, and it also reports on other intramolecular or intermolecular H-bonding interactions [25]. Furthermore, the fluorescence is not affected by $\mathrm{pH}$ or by the presence of phosphate anions, making it compatible with different biological contexts, but is strongly quenched by $\mathrm{Cl}^{-}$anions. These can be used to probe the effects of different structures and/or environments surrounding the probe (folded vs unfolded, bulk solution vs membrane interaction etc.) [22]. It shows a mono-exponential decay in water, $(\tau \sim 7 \mathrm{~ns})$, but this can become non-exponential when it is inserted into proteins exhibiting different conformations [25]. We have attempted to exploit these features to see if $\mathrm{F}_{\mathrm{CN}}$ could report on its local environment when in different positions of the LL-37 or RL-37 chains and so provide additional information on the conformational transitions occurring when the molecules pass from an aqueous to a membrane environment, and how this is affected by oligomerization.

\section{1) Fluorescence intensities and lifetimes of $F_{C N}$ analogue peptides in aqueous solution}

The fluorescence spectrum of the reference peptide $\mathrm{GF}_{\mathrm{CN}} \mathrm{KG}$ in aqueous solution is shown in Figure 2A. In agreement with the literature, phosphate ions did not affect the emission intensity, while increasing concentrations of $\mathrm{Cl}^{-}$significantly reduced it [22]. The presence of negatively charged liposomes, $\left(\mathrm{LUV}^{(-)}, \mathrm{PG} / \mathrm{dPG}, 95: 5\right)$, or neutral ones $\left(\mathrm{LUV}^{(0)}, \mathrm{PC} / \mathrm{SM} / \mathrm{Ch}, 40: 40: 20\right)$ also had no effect on fluorescence, confirming that the reference peptide does not interact with them. The emission intensities of LL-37 or RL-37 analogues in pure water solution, normalized to that of $\mathrm{GF}_{\mathrm{CN}} \mathrm{KG}$, are shown in Figure 2B. Under these conditions both peptides are in a random coil conformation, as clearly shown by CD spectroscopy (supplementary material Figure S1 D and E). As expected, analogues with $\mathrm{F}_{\mathrm{CN}}$ at positions 17 or 27 behaved similarly to $\mathrm{GF}_{\mathrm{CN}} \mathrm{KG}$, indicating that the probe is completely exposed to water. A significantly reduced fluorescence intensity was instead observed for analogues with $\mathrm{F}_{\mathrm{CN}}$ in position 5, similarly to the $\mathrm{GF}_{\mathrm{CN}} \mathrm{FKG}$ reference peptide (Figure 2B), whose emission intensity is only about $80 \%$ that of $\mathrm{GF}_{\mathrm{CN}} \mathrm{KG}$. A Phe residue adjacent to, or spatially close to the probe residue can thus significantly affect it fluorescence, an unexpected effect that to our knowledge has not been considered in previous studies [23].

The structure of $\mathrm{GF}_{\mathrm{CN}} \mathrm{FK}$ was modelled in a fully extended or $\alpha$-helical conformation, using the Sybyl 7.3 suite, and the distance between the centers of the two rings was determined respectively to be $7.2 \pm 1$ and $8.6 \pm 1.3 \AA$. This could place the two residues within resonance energy-transfer distance, as has been reported to occur with Tyr or Trp [33,34], except that there is very little spectral overlap between the $\mathrm{F}_{\mathrm{CN}}$ emission and Phe absorption (Figure 2C). The emission intensity 
decrease in $\mathrm{GF}_{\mathrm{CN}} \mathrm{FKG}$ with respect to $\mathrm{GF}_{\mathrm{CN}} \mathrm{FKG}$ would suggest a Forster's distance of only $6.5 \pm$ $1.5 \AA$ for the Phe $\mathrm{CN}_{\mathrm{CN}}$ donor/ Phe acceptor pair, consistent with a low spectral overlap. Another possibility is that quenching occurs by photoinduced electron transfer (PET), which is known to occur with cyanoaromatic groups acting as acceptors, and alkylbenzene groups as electron donors, and can act over several bonds, including peptide bonds [35]. However, Phe is considered to be a redox-inactive side-chain, making this unlikely [35]. These aspects need to be taken into consideration when using $\mathrm{F}_{\mathrm{CN}}$ as a probe, and require further clarification.

A problem with steady-state fluorescence as an indicator of the environment surrounding the $\mathrm{F}_{\mathrm{CN}}$ probe is that it measures the average of the fluorescence intensity of the sample. In this respect it is more useful to monitor the emission decay kinetics, as these can report on coexisting probe populations. The fluorescence lifetime decay of $\mathrm{GF}_{\mathrm{CN}} \mathrm{KG}$ in pure water (Figure 3A) deviated from mono-exponential decay, but showed a reasonable bi-exponential fit (see residuals in Fig. 3B \& $\mathbf{C}$ ), where the more abundant component $\left(\tau_{1}=8.1 \mathrm{~ns}, \mathrm{~A}_{1}=85 \%\right)$ is consistent with $\mathrm{CN}$ groups being totally exposed to water molecules [25]. The other faster component $\left(\tau_{2}=3.2 \mathrm{~ns}, \mathrm{~A}_{2}=15 \%\right)$ might be due to quenching by residual chloride counter ions deriving from the peptide work-up process (see below). The fluorescence decay curves for $\mathrm{F}_{\mathrm{CN}}{ }^{17}$ in LL-37, and $\mathrm{F}_{\mathrm{CN}}{ }^{27}$ in either LL-37 or RL-37, were effectively superimposable to those of $\mathrm{GF}_{\mathrm{CN}} \mathrm{KG}$, and dominated by a similar longer-lived component (Table 2). For the $\mathrm{GF}_{\mathrm{CN}} \mathrm{FKG}$ reference peptide, $\tau_{1}$ was shorter than for $\mathrm{GF}_{\mathrm{CN}} \mathrm{KG}\left(\tau_{1}=\right.$ $6.3 \mathrm{~ns}, \mathrm{~A}_{1}=80 \%$ ), as expected from the steady state fluorescence behaviour, likely due to some form of quenching by the adjacent $\mathrm{F}^{6}$. The decay curves for $\mathrm{F}_{\mathrm{CN}}{ }^{5}$ in the LL-37 and RL-37 analogues were also essentially superimposable to that of $\mathrm{GF}_{\mathrm{CN}} \mathrm{FKG}$. In summary, all probe side-chains are fully exposed to water when the peptides are in a random-coil conformation, and some may be subject to quenching by residual chloride counterions.

\section{2) Effect of salt ions on structure and fluorescence}

It is well known that LL-37 adopts a helical conformation in the presence of appropriate salt ions, at neutral $\mathrm{pH}[3,12,36]$. This is likely due to the establishment of a network of intermolecular and intramolecular salt-bridges between appropriately placed anionic and cationic residues, which is lacking in rhesus RL-37. Self-assembly of LL-37 is also driven by the necessity to shield the hydrophobic sector of the amphipathic helix from the aqueous environment. This effect is observable in CD spectra already in 10mM SPB (Figure S1 A), where it was estimated to have a helix content of about $20 \%$ based on the ellipticity value at $222 \mathrm{~nm}$ [27], while RL-37 remains in random coil conformation. LL-37 is thus in equilibrium between an unstructured/monomeric and structured/oligomeric form. In the presence of the strongly helix-inducing solvent trifluoroethanol (TFE) the peptide reaches a maximum helix content of 55-60\% [13,37], likely limited by end effects and distortions in the helix. Therefore a helical content of about $20 \%$ in SPB would indicate a roughly 1:2 structured/disordered peptide ratio.

In $10 \mathrm{mM} \mathrm{SPB}$, the decay lifetime $\left(\tau_{1}\right)$ of the reference peptides is not greatly altered with respect to pure water (Figure 4). The same was observed for RL-37 analogues, for which no structural transition was observed in this buffer, decays were superimposable on those of the reference peptides (Table 3). For the LL-37( $\left.\mathrm{F}_{\mathrm{CN}}\right)^{17}$ and $\left(\mathrm{F}_{\mathrm{CN}}\right)^{27}$ analogues, two components of equal amplitude but with significantly different lifetimes emerged. Thus, the transition to a helical conformation and oligomerization changes the environment around these probe residues in a manner that affects their emission. LL-37( $\left.\mathrm{F}_{\mathrm{CN}}{ }^{5}\right)$ instead behaves similarly as in aqueous solution, and its emission decay resembles that of $\mathrm{GF}_{\mathrm{CN}} \mathrm{FKG}$, so it appears to be in a less affected region.

Raising the phosphate buffer concentration to $100 \mathrm{mM}$, increased the helix content to $~ 35 \%$ for LL-37 (Figure S1A, roughly 1:1 structured/disordered peptide ratio), while still not inducing a helical conformation in RL-37. The steady state fluorescence intensities, normalized to that of 
$\mathrm{GF}_{\mathrm{CN}} \mathrm{KG}$, are shown in Figure 5. Only LL-37( $\left.\mathrm{F}_{\mathrm{CN}}\right)^{27}$ shows a significant variation in steady state emission. The fluorescence lifetimes for $\mathrm{GF}_{\mathrm{CN}} \mathrm{KG}$ and $\mathrm{GF}_{\mathrm{CN}} \mathrm{FKG}$ were unaffected by phosphate or sodium ions, in agreement with the literature [22] (Table 4). A similar result was observed for RL$37\left(\mathrm{~F}_{\mathrm{CN}}\right)^{5}$ and $\left(\mathrm{F}_{\mathrm{CN}}\right)^{27}$ analogues. All three LL-37 analogues, instead, showed long-lived components with $\sim 50 \%$ abundance (Table 4), and the central $\mathrm{F}_{\mathrm{CN}}{ }^{17}$, in particular, has a markedly increased lifetime (17 ns). The other component observed (4.8 - $6.2 \mathrm{~ns}$ ) has an intermediate lifetime to that of the longer and shorter-lived components observed in pure water, so could be due to probe residues exposed to the aqueous environment (see Table 2). We did not attempt to verify this using a three exponential fit to avoid over interpreting the data, given also that the $\chi^{2}$ values of the bi-exponential fitting model were acceptable (Table 4). The effect on $\mathrm{F}_{\mathrm{CN}}{ }^{5}$ was less marked than for $\mathrm{F}_{\mathrm{CN}}{ }^{17}$ or $\mathrm{F}_{\mathrm{CN}}{ }^{27}$, again suggesting that helical structuring involves the $\mathrm{N}$-terminus less than the central region, in agreement with previous structural studies [11].

The fact that changes in peptide conformation could result in an environment around the $\mathrm{F}_{\mathrm{CN}}$ probe that so markedly increases its lifetime, has to our knowledge, not been previously reported. Most literature reports, however, describe either variation in steady state fluorescence, which would miss this effect, or FRET experiments in which the probe is quenched by sequentially or spatially close Trp residues [24], not allowing the long-lived component to emerge. The extended lifetime could result from probe residues forming strong inter- or intra-molecular H-bonds with appropriately placed donor moieties, when the peptide adopts a helical conformation and/or oligomerises. Just being buried in the hydrophobic core of an oligomer would not provide an explanation, as in the case of $\mathrm{F}_{\mathrm{CN}}$, the emission increases with the H-bonding capacity of the solvent, and is not favoured by an aprotic environment [25].

A peculiar aspect of these studies is the apparent discrepancy between steady-state and timeresolved data. The presence of a sizeable component with significantly increased lifetime should result in a significantly increased steady-state fluorescence intensity in the presence of phosphate buffer, whereas this is unaffected or even decreased with respect to aqueous solution (Figure 5A and $\mathbf{B}$ ). This may be an indication that the time-correlated single photon counting method used is missing a very fast component within the dead time of the instrument. This could occur if, on oligomerization, some of the probe side-chains were placed very close to a quencher moiety. In particular, a parallel arrangement of peptide chains might lead to quenching by vicinal $\mathrm{F}$ or $\mathrm{F}_{\mathrm{CN}}$ groups. Under this scenario, part of the probe side-chains would engage in strong H-bonding with a nearby donor, significantly increasing its lifetime, some would remain exposed to the solvent environment, and some would pack very close to a group capable of efficient quenching. This aspect needs further study.

To gain further insight into how the long-lived component correlates with structural characteristics, we compared the behaviour of LL-37( $\left.\mathrm{F}_{\mathrm{CN}}\right)^{17}$ in the presence of up to $50 \% \mathrm{TFE}$ or in acetonitrile. In the former condition the CD spectrum of LL-37 ( $\theta_{222}$ value $)$ indicated $>50 \%$ helix content, and its shape $\left(\theta_{280} / \theta_{222}>1\right)$ suggested an isolated helix [38]. In the presence of acetonitrile, the $\theta_{280} / \theta_{222}$ ratio inverts, suggesting that the peptide is in a stacked form (Figure 6A). Addition of TFE $(5-50 \%)$ increased the lifetime of LL-37( $\left.\mathrm{F}_{\mathrm{CN}}\right)^{17}$ progressively, while not affecting the lifetime of $\mathrm{GF}_{\mathrm{CN}} \mathrm{KG}$. Note that this solvent has a Kamlett-Taft $\alpha$ parameter higher than water (see Supplementary materials Table 1S), so that it could increase the fluorescence lifetime independently of its effect on conformation [25]. However, the increase in lifetime seems to follow the \% helix content more than \%TFE (Figure 6B), suggesting it is due to the peptide's conformation rather than the presence of TFE. On the other hand, in the presence of acetonitrile that has a significantly lower Kamlett-Taft $\alpha$ parameter than water, $\mathrm{GF}_{\mathrm{CN}} \mathrm{KG}$ showed a reduced $\tau_{1}$ and increased abundance of the $\tau_{2}$ (shorter-lived components), as expected [25]. Nonetheless, for LL$37\left(\mathrm{~F}_{\mathrm{CN}}\right)^{17} \tau_{1}$ is increased to $12.7 \mathrm{~ns}$. Taken together, these data indicate that on helix formation both 
inter-molecular and intra-molecular H-bonds involving some probe side-chains lead to a population with increased lifetime.

\section{3) Effect of peptide conformation and oligomerization on quenching by chloride ions.}

The differentially increased lifetimes of $\mathrm{F}_{\mathrm{CN}}$ under structure-inducing conditions prompted us to investigate probe side-chain exposure using chloride anions as quenchers $[22,35]$. This however has to take into account that increasing salt concentrations favour helix formation in LL-37 (a helical content of up to $25 \%$ was observed at $200 \mathrm{mM} \mathrm{NaCl}$ ) (Figure S1 E and F). The steady state emission spectra for the two reference peptides and LL-37 analogues under different conditions are shown in Figure 7A and $\mathbf{B}$ (and in supplementary materials Fig S3). The steady-state emission intensity of LL-37 analogues is decreased in a concentration-dependent manner by $\mathrm{Cl}^{-}$in a similar manner to that of the reference peptides, indicating dynamic quenching.

Despite a significant helix content for LL-37 the $\mathrm{F}_{\mathrm{CN}}$ side-chains in all three analogue peptides are not shielded from quenching (Figure 7C), so that folding and oligomerization do not place them in a protected environment. This supports that the suggestion that the component with increased lifetime observed on oligomerization is due to increased H-bonding [25], rather than to burial into an isolated core.

In agreement with the steady state emission, both reference peptides showed an emission lifetime significantly decreased down to $\sim 2 \mathrm{~ns}$ in $200 \mathrm{mM} \mathrm{NaCl}$ (Figure 7D). The same was observed for RL-37 analogues (not shown), consistent with its completely unfolded, monomeric form under these conditions (see Figure S1D). For LL-37 analogues, instead, a long-lived component emerged as the helical content increased with the $\mathrm{Cl}^{-}$concentration, but with a low amplitude at $>50 \mathrm{mM} \mathrm{Cl}^{-}$anions. Thus, for the $\mathrm{F}_{\mathrm{CN}}{ }^{17}$ analogue, $\tau_{2}$ was essentially unchanged at $3 \mathrm{~ns}$ at $200 \mathrm{~mm} \mathrm{NaCl}$ but its amplitude was $>80 \%$, while $\tau_{1}$ increased to almost $17 \mathrm{~ns}$ in the presence of $\mathrm{Cl}^{-}$(similar to those in $100 \mathrm{mM} \mathrm{SPB}$ ) but with amplitude $<20 \%$ (Figure 7E and 7F). The $\mathrm{F}_{\mathrm{CN}}{ }^{27}$ and $\mathrm{F}_{\mathrm{CN}}{ }^{5}$ analogues showed a similar trend but with less marked increases in the lifetime of the long-lived component (to 12 and $8 \mathrm{~ns}$ respectively, also similar to $100 \mathrm{mM} \mathrm{SPB}$ ).

The transition to a helical structure therefore does afford a partial protection of the fluorophore since a longer-lived component was observed in the presence of the quencher, but is limited to a minor probe population. Vice-versa, the lifetime of the short-lived component observed in the presence of $200 \mathrm{mM} \mathrm{Cl}^{-}$was similar to that of the short-lived component in water, but with much higher amplitude. This lends support to the possible role of residual chloride ions in quenching $\mathrm{F}_{\mathrm{CN}}$ in aqueous solution [22]. These results are again best interpreted in terms of an equilibrium between unfolded and helical/oligomeric states for LL-37, in which a portion of probe side-chains (particularly in positions 17 and 27) enter into a core region where they form stable H-bonds and are thereby also protected from the quencher.

\section{4) Peptide-membrane interaction studies}

Previous studies have shown that LL-37 causes a different type of lesions in biological membranes than the rhesus RL-37, as their different folding/oligomerisation behaviour likely leads to a different initial interaction with the membrane, affecting their subsequent mode of action $[3,13,15]$. To confirm this, and to gain more information on this process at the molecular level, we investigated emission of our analogues in the presence of model membranes.

For this purpose, negatively charged $\mathrm{LUV}^{(-)}(\mathrm{PG} / \mathrm{dPG}, 95: 5)$ were used as a model for bacterial membranes, and neutral LUV ${ }^{(0)}$ (PC/SM/Ch, 40:40:20) as a model for host cell membrane. In both cases, a peptide/lipid (P/L) ratio of $1 / 20$ was used. CD spectra showed that in presence of $\mathrm{LUV}^{(-)}$ both peptides folded into an $\alpha$-helix, but only LL-37 did so when in contact with the LUV ${ }^{(0)}$ (Figure S1 B and C). The emisson spectra and lifetimes of peptides were measured first in LUV suspensions in $100 \mathrm{mM} \mathrm{SPB}$, and subsequently $\mathrm{NaCl}$ was added to a final concentration of $200 \mathrm{mM}$. 
The aim was to determine if on membrane interaction $i$ ) the $\mathrm{F}_{\mathrm{CN}}$ side-chains were inserted into the relatively aprotic environment of the lipid layer, thus reducing emission in the presence of LUVs; $i i$ ) if this protected $\mathrm{F}_{\mathrm{CN}}$ side-chains from $\mathrm{Cl}^{-}$quenching, so that the emission should remain unaltered on adition of $\mathrm{NaCl}$, and $\mathrm{iii}$ ) how the oligomeric nature of LL-37 was affected.

The emission intensity and lifetime of reference peptides was unaffected in the presence of LUVs, as they do not interact with membranes (Figure 8) and was strongly quenched by $\mathrm{Cl}^{-}$(Table 6). For the LL-37 analogues, the fluorescence intensity was reduced by $40-70 \%$ in the presence of LUV $^{(-)}$(Figure 8, top panel), consistent with insertion of the probe side-chains into the aprotic bilayer environment. The more central $\mathrm{F}_{\mathrm{CN}}{ }^{17}$ showed the strongest reduction (70\%) followed by $\mathrm{F}_{\mathrm{CN}}{ }^{5}$ (supplementary material, Figure S4). Addition of $200 \mathrm{mM} \mathrm{Cl}^{-}$had no further effect. Two well distinguished lifetimes were observed for all three analogues, with $\tau_{1}=9-10 \mathrm{~ns}(60-70 \%)$ and $\tau_{2}=2$ 3 ns (30-40\%) (Table 6), and addition of $\mathrm{Cl}$ - confirmed $\mathrm{F}_{\mathrm{CN}}$ shielding as $\tau_{1}$ was only slightly affected. RL-37 analogues behaved similarly (Figure 8, top panel and Table 6), suggesting that both peptides interact strongly with anionic membranes, being inserted in the lipid bilayer in helical conformations.

As to what generates the two components with different lifetime under these conditions, it is proposed that amphipathic, helical AMPs interact with the surface of bacterial membranes, lying parallel to it, and that they insert their hydrophobic sectors into the bilayer, floating on its surface in a log-like manner. Insertion of $\mathrm{F}_{\mathrm{CN}}$ into the aprotic environment could explain the increased abundance short-lived component, but as the long-lived component still dominates, a significant proportion of probe side-chains must still be capable H-bonding interactions [25]. For RL-37, which is monomeric, this could be due either to intra-molecular H-bonds, or those with phospholipid headgroups. For LL-37 some level of oligomerization persists in the membrane [3], so that intermolecular H-bonding could also occur. However, the fact that the lifetime is less than that observed in $100 \mathrm{mM}$ SPB ( 10 vs $17.4 \mathrm{~ns}$, Table 4), suggests a looser type of aggregation in the presence of anionic membrane, consistent with the previous photochemical studies [3].

The steady-state emission spectra for LL-37 analogues decreased less in the presence of neutral with respect to anionic LUVs, but the effect was still significant (Figure 8, bottom panel), indicating some form of interaction with the membrane that alters the environment of $\mathrm{F}_{\mathrm{CN}}$ groups (Figure S5 in supplementary material). In this case, however, addition of $200 \mathrm{mM} \mathrm{Cl}^{-}$caused a further decrease. Both these observations are consistent with less insertion into the lipid bilayer. Furthermore, emission lifetimes were also less affected in the presence of $\operatorname{LUV}^{(0)}$ (Table 6), pointing to a surface location of LL-37 oligomers, which afford some protection form quenching by $\mathrm{Cl}^{-}$. RL-37 emission intensity and lifetimes were unaffected by neutral LUVs, and not shielded from $\mathrm{Cl}^{-}$quenching (Table 6), suggesting a much looser interaction with this type of membrane, in agreement with CD spectra (Figure S1 C).

The stretching vibrations of the $\mathrm{C} \equiv \mathrm{N}$ group are very sensitive to its environment, and result in absorption in a relatively transparent region of the typical protein IR spectrum, making $\mathrm{F}_{\mathrm{CN}}$ an excellent IR probe $[17,26]$. We have previously succesfully used this to study the interaction of a helical stretch in the human defensin hBD3 with biological membranes [28]. Transmission IR measurements for peptides were carried out with concentrated solutions $(2 \mathrm{mM})$ and very short path lengths, due to the strong IR signal of water. The vibrational band for $\mathrm{F}_{\mathrm{CN}}$ residues in both LL-37 and RL-37 analogues (see Table 7) were in the reported range for an aqueous environment $(v=$ $2235 \pm 0.5 \mathrm{~cm}^{-1}, \delta v=13 \pm 1 \mathrm{~cm}^{-1}$ ) [26]. To observe the effect of membrane interactions, ATRFTIR spectra were measured after the peptides had been flowed over supported membrane multibilayers, laid directly over the germanium crystal in a custom made cell [28] (to an estimated P/L ratio of about $1 / 20$, see materials and methods section). The $C \equiv N$ stretching vibration in membranelike environments (AOT reverse micelles), is reported to shift to $2229.5 \pm 1 \mathrm{~cm}^{-1}$ ( $\delta v=7.3 \pm 0.4$ $\mathrm{cm}^{-1}$ ) [40]. In the presence of PG/dPG supported bilayers, we observed a similar shift for both LL- 
37 and RL-37 analogues (Table 7). These results, consistent with those of fluorescence studies, confirm interaction of both peptides with anionic membranes, leading to insertion of the probe sidechains into the lipid bilayer. In this case, significant differences with respect to the position of the probe side-chain $(5,17$ or 27$)$ were not observed.

The CN stretching band was also blue-shifted in presence of neutral lipid multilayers (PC/SM/Ch) (Table 7). The broader bandwidth with respect to anionic LUVs $\left(>10 \mathrm{~cm}^{-1} \mathrm{vs} \approx 8 \mathrm{~cm}^{-}\right.$ $\left.{ }^{1}\right)$ may support a less inserted state and more heterogeneous environment for the neutral membrane, in line with fluorescence results. In this case, however, a comparable blue-shift was observed for LL-37 and RL-37, in contrast with fluorescence or CD studies, which indicated little interaction of RL-37 with neutral LUVs. The explanation may derive from the ATR setup, which detects molecules within the evanescent field of the IR radiation close to the crystal surface and within the membrane bilayer, while failing to detect those more on the surface. In other words, it samples small amounts of peptide that partition into the membrane, which are instead missed by CD or fluorescence spectroscopy that sample the whole populations. This aspect of the $F_{C N}$ probe is therefore quite useful, as it indicates that even for RL-37, some peptide molecules do partition into neutral membranes. In this respect, it should be noted that in studies on the biological effects of Rl37 on eukaryotic cells (e.g. haemolysis, LDH release or PI permeabilization), while it is generally less lytic than LL-37 at low concentrations, the effects are comparable at higher ones [3, 13, 15, 38]. This implies that there must be some form of interaction, which eventually leads to lysis of neutral membranes, by RL-37 as well as LL-37.

\section{5) Surface plasmon resonance studies of peptide self-association and membrane interaction}

We have attempted to use a Biacore setup to see if we could i) observe self-assembly of free flowing LL-37 molecules (analyte) with LL-37 molecules supported on the sensor surface (ligand), and ii) obtain more information on the modes of binding of LL-37 or RL-37 with anionic and neutral membranes.

In the first case, LL-37 molecules were anchored to the carboxyl dextrans covering the surface of a CM5 chip, via formation of amide bonds with the N-terminal or lysine amines. Self-association was then investigated by flowing LL-37 in solution over the chip surface, at different concentrations and $\mathrm{pH}$ values. In the second case, $\mathrm{LUV}^{(-)}$(DPPC/DPPG 4:1) or LUV ${ }^{(0)}$ (only DPPC) were anchored to lipid modified dextrans in the L1 chip, before flowing peptide solutions over the chip surface.

Peptide self-assembly: Increasing concentrations of an LL-37 solution ( $4-64 \mu \mathrm{M})$ in mildly basic conditions, where the peptide is in the helical conformation (as verified by $\mathrm{CD}$, not shown), were flowed over the chip surface. Sensorgrams indicate only a very moderated, linear increase in response units (RU) up to $16 \mu \mathrm{M}$ of analyte, consistent with a weak and unspecific interaction, but at $32 \mu \mathrm{M}$ there was a jump to almost $1000 \mathrm{RU}$, and a further increase to over $4000 \mathrm{RU}$ at $64 \mu \mathrm{M}$ (Figure 9A). It was not possible to determine an affinity constant with this behaviour. The binding was rapid and reversible, suggesting a massive level of reversible aggregation onto the surfacebound LL-37 molecules.

A quite different behaviour was observed when the analyte was flowed in sodium acetate buffer ( $\mathrm{pH}$ 5) under which conditions the peptide is unstructured (as verified by $\mathrm{CD}$, not shown), as acidic $\mathrm{pH}$ disrupts intramolecular salt-bridging. In this case, a more homogeneous, concentrationdependent increase of the RU signal was observed, up to about $500 \mathrm{RU}$ at $64 \mu \mathrm{M}$ (Figure 9B), and was possible to calculate the affinity constant, $K_{D}=6 \times 10^{-7} \mathrm{M}$. Binding was less reversible, with a significant signal also after the washing step, indicating a slower off rate. These results might be explained by a more intimate type of electrostatic interaction between unstructured free-flowing and anchored peptide chains. 
Peptide-membrane interactions were studied using freshly prepared LUVs flowed onto the L1 chip covered with lipid modified dextrans, to which they anchor. The conditions under which the chip were prepared, and the phospholipid composition of the LUVs, were such that these were likely to remain intact on anchoring [30]. The interaction of LL-37 and RL-37 with supported anionic LUVs are shown in Figure 9C \& $\mathbf{D}$, and can be correlated with the secondary structure in the presence of the same type of LUVs, as determined using CD spectroscopy (Figure S1 B). The sensorgrams indicate that the two peptides have somewhat different modes of interaction with membranes, as the RU increase dramatically for LL-37, over a threshold concentration of about $2 \mu \mathrm{M}$, and this may be due to self-association or oligomer insertion at the membrane surface. The affinity constant could thus not be calculated. For RL-37 the variation in RU has a more linear dependence on the concentration, allowing calculating a $K_{D}$ of about $5 \times 10^{-6} \mathrm{M}$.

Unexpectedly, a quite similar behaviour was observed for chips covered with $\operatorname{LUV}^{(0)}$, for both LL-37 and RL-37 (Figure 9E and F). Thus, even though CD spectra indicate that neutral LUVs do not induce large scale structure formation in the latter peptide, SPR experiments would appear to confirm results from IR experiments in suggesting that some peptide molecules can interact with this type of membrane.

\section{6) Flow cytometric studies of bacterial membrane permeabilization}

Our results suggest that the particular behaviour of LL-37 and RL-37 in solution, disordered or helical conformation resulting in a monomeric or oligomeric form, strongly affects their interaction with the membranes. We have previously proposed that this then leads to distinct types of membrane permeabilization, based on AFM experiments with supported anionic model membranes [15], or on the size of fluorescent dyes that could transit through the cytoplasmic membrane of bacteria, as determined by flow cytometry [3]. We have refined this technique by using probe dyes of different sizes, ranging from propidium iodide (PI, $1.6 \mathrm{~nm}$ diameter), to fluoresceinated dextran particles of increasing sizes (FITC-Dx of 4, 10, and $40 \mathrm{kDa}$ corresponding 2.8, 4.6, and $9 \mathrm{~nm}$ diameters, respectively, as reported in Sigma technical sheets).

This technique allowed us to distinguish more precisely the dimension of the lesions caused by the two peptides to the S. aureus ATCC25923 bacterial membrane, in order to elucidate differences in the mode of action of these two structurally diverse peptides. Bacterial cells were incubated for 60 min with increasing peptide concentrations. The percentage of permeabilized cell was measured in flow cytometry from the fluorescence signal obtained when the dyes enter into the cell. Results for simultaneous exposure to PI and FD40 (FITC-DX $40 \mathrm{kDa}$ ) are shown as biparametric dot plots in Figure 10A and B. LL-37 caused lesions of a small size at $2 \mu \mathrm{M}$, as PI could enter in $\sim 30 \%$ of the bacterial cells, but all cells were negative for FD40. However, increasing the concentration to 4 $\mu \mathrm{M}$ resulted in larger lesions that allowed FD40 molecules $(\varnothing=9 \mathrm{~nm})$ to enter about $30 \%$ of cells (Figure 10A and D). The effect was even more evident at $8 \mu \mathrm{M}$ of peptide concentration, where $80 \%$ of the cells were permeabilized to FD40. Furthermore, it occurred within 30 min with little further change at 60 min (not shown). RL-37 was considerably more efficient than LL-37 in permeabilizing the S. aureus membrane to PI, with $100 \%$ of PI positive at just $2 \mu \mathrm{M}$, but the size of the lesion remained unaltered even at $8 \mu \mathrm{M}$ peptide as no signal for internalized FD40 was detected (Figure 10B and D). We then tested the uptake of FITC-Dx of smaller sizes (4 and $10 \mathrm{kDa})$, which made the different modes of membrane permeabilization even more evident (Figure 10E). S. aureus cells treated with $8 \mu \mathrm{M}$ RL-37 did not allow the uptake of probes bigger than PI (e.g. FD4 was internalized in less than $5 \%$ of cells), so that lesions were must be small. Bacteria treated with the same concentration of LL37 instead allowed the uptake of probes in 40-60\% of cells, in a size dependent manner. Results suggest that a significant proportion of the pores/lesions that form are large enough to allow the passage of even the bulkier probe. 
Thus, in agreement with previous studies on model membranes [15], we confirmed that RL-37 disrupts the bacterial membrane with a higher efficiency than LL-37. This may in part be due to a lower tendency to be sequestered by medium and/or bacterial outer cell wall components (e.g. peptidoglycan, wall teichoic and lipoteichoic acids). The type of lesions that it causes to the cytoplasmic membrane are small in size $(\varnothing<2.8 \mathrm{~nm})$, so that fewer peptide molecules may be sufficient to exert a permeabilizing effect. Oligomeric LL-37 requires higher concentrations to become toxic to $S$. aureus cells, in part due to a higher tendency to be sequestered, and forms larger pores or lesions $(\varnothing \sim 9 \mathrm{~nm}$ ) that may require the co-operation of several peptide molecules. In AFM experiments, distinct channels of approximately these dimensions were in fact observed for supported membranes exposed to LL-37, while those exposed to RL-37 had a more disordered 'spongy' appearance [15]. We interpret this as RL-37 causing a "detergent-like" damage to the membrane that allows PI to leak in but not larger molecules.

\section{Conclusion.}

Our comparison of the human and macaque cathelicidin orthologues leads to a proposed model for their modes of action that is summarized in Scheme 1, which attempts to explain how conformational and aggregational states can affect biological roles. LL-37, in solution, is in equilibrium between different structural/oligomeric forms, which correlate with the observed lifetimes of the intrinsic $\mathrm{F}_{\mathrm{CN}}$ probes. The unfolded/monomeric peptide $(\tau \sim 8 \mathrm{~ns})$, is predominant in aqueous solution, where the probe is fully exposed to the environment and subject to efficient $\mathrm{Cl}^{-}$ quenching, which significantly reduces the lifetime $(\tau \sim 3 \mathrm{~ns})$. The folded, helical form occurs significantly in the presence of physiological salt concentrations at neutral $\mathrm{pH}$ obliging the peptide to oligomerise to remove its hydrophobic face from the aqueous environment (Scheme $1 \mathbf{0}$ ). This is favoured by intramolecular and intermolecular salt-bridges between appropriately positioned basic and acidic residues, and cannot occur for RL-37, which remains in the F-form. It results in increased $\mathrm{H}$-bonding to the $\mathrm{F}_{\mathrm{CN}}$ probe resulting in a component with markedly increased lifetime ( $\tau$ $\sim 17 \mathrm{~ns}$ ), especially for the central $\mathrm{F}_{\mathrm{CN}}{ }^{17}$. This effect is due to oligomerization rather than just helical folding, as shown by a smaller effect of TFE (2) than acetonitrile (3). LL-37 and RL-37 approach the bacterial membrane respectively monomeric and oligomeric forms, which affects the subsequent interaction and mode of permeabilization: RL-37 interacts with the membrane surface as a monomer (4), then adopts a helical structure and inserts into the bilayer as a monomer. LL-37 interacts with the surface as an oligomer (5). This has a marked effect on the antimicrobial activity, as the monomeric peptide is less subject to sequestration by serum or medium components, or components of the bacterial outer cell wall. For this reason RL-37 is more a potent and broadspectrum antibiotic than LL-37.

On interaction with the membrane surface, LL-37 can form sizeable channels compatible with toroidal pores, that allow larger molecules through $(\boldsymbol{6})$, although it may also in part disassociate to an inserted form similar to that of RL-37 ( $)$ ). RL-37 can instead only form small, heterogeneous lesions allowing transit of only small molecules through the membrane (8). In a previous study [15], we have reported that the small, heterogeneous lesions caused by RL-37 occur very rapidly and lead to membrane depolarization, whereas the larger channels induced by LL-37 lead to a slower depolarization. By analogy to other helical AMPs such as magainin, RL-37 might be acting by a detergent or carpet-like mechanism. However, this is proposed to eventually lead to extensive micellization of the membrane [41], with quite large lesions forming, while we did not observe such lesions after exposure to $8 \mu \mathrm{M}$ RL-37 for 1 hour, so if it occurs it would seem to be a slow process, possibly due to the stabilizing effect of the peptidoglycan layer.

Whether the peptides acts singly, via a detergent-like effect, or in a more concerted manner, via formation of toroidal pores, they remain parallel to the membrane surface, and are only partly inserted into the lipid bilayer. This gives rise to a similar pattern of short-lived ( $\left.\tau_{2} \sim 3 \mathrm{~ns}\right)$ and long- 
lived ( $\tau_{1} \sim 10 \mathrm{~ns}$ ) fluorescence decay components for LL-37 or RL-37 in the presence of anionic LUVs, due respectively to insertion of the probe side-chains into aprotic the lipid bilayer or $\mathrm{H}$ bonding with appropriate donors in the headgroup region.

In the presence of neutral membranes LL-37 appears to be capable of interacting with the membrane surface as an oligomer (5) but with a smaller proportion of molecules inserted into the bilayer. RL-37 has a lower capacity for this type of interaction (possibly deriving from its monomeric state) but it does occur, as indicated by IR and Biacore experiments. It is possible that the oligomeric form of LL-37, in which the peptide is already in a helical conformation, favours its interaction with host cell membranes, allowing it to better exert its pleiotropic effects on different types of host cells [42].

\section{Acknowledgements}

We thank Prof. Thomas Kiefhaber for the opportunity given to perform the measurements using time-resolved fluorescence instrument in his laboratory and for the scientific discussions and advice. We also thank Prof. J.M. Kelly for his sound advice and suggestions.

\section{Author contribution}

Daniela Xhindoli performed or participated in all experiments. Francesca Morgera was responsible for ATR-FTIR experiments. Ursula Zinth contributed for interpretation of the time-resolved fluorescence data. Sabrina Pacor was responsible for flow cytometry experiments. Daniela Xhindoli and Alessandro Tossi designed experiments, interpreted the results and wrote the manuscript.

\section{References}

1 Sochacki, K. A., Barns, K. J., Bucki, R. and Weisshaar, J. C. (2011) Real-time attack on single Escherichia coli cells by the human antimicrobial peptide LL-37. Proc. Natl. Acad. Sci. U.S.A. 108, E77-81

2 Barns, K. J. and Weisshaar, J. C. (2013) Real-time attack of LL-37 on single Bacillus subtilis cells. Biochim. Biophys. Acta. 1828, 1511-1520

3 Xhindoli, D., Pacor, S., Guida, F., Antcheva, N. and Tossi, A. (2014) Native oligomerization determines the mode of action and biological activities of human cathelicidin LL-37. Biochem. J. 457, 263-275

4 Brown, K. L., Poon, G. F., Birkenhead, D., Pena, O. M., Falsafi, R., Dahlgren, C., Karlsson, A., Bylund, J., Hancock, R. E. and Johnson, P. (2011) Host defense peptide LL-37 selectively reduces proinflammatory macrophage responses. J. Immunol. 186, 5497-5505

5 Ruan, Y., Shen, T., Wang, Y., Hou, M., Li, J. and Sun, T. (2013) Antimicrobial peptide LL37 attenuates LTA induced inflammatory effect in macrophages. Inter. Immunopharmacol. 15, 575580

6 Mookherjee, N., Brown, K. L., Bowdish, D. M., Doria, S., Falsafi, R., Hokamp, K., Roche, F. M., Mu, R., Doho, G. H., Pistolic, J., Powers, J. P., Bryan, J., Brinkman, F. S. and Hancock, R. E. (2006) Modulation of the TLR-mediated inflammatory response by the endogenous human host defense peptide LL-37. J. Immunol. 176, 2455-2464

7 Fu, H., Karlsson, J., Bylund, J., Movitz, C., Karlsson, A. and Dahlgren, C. (2006) Ligand recognition and activation of formyl peptide receptors in neutrophils. J. Leukoc. Biol. 79, 247-256

8 Heilborn, J. D., Nilsson, M. F., Kratz, G., Weber, G., Sorensen, O., Borregaard, N. and Stahle-Backdahl, M. (2003) The cathelicidin anti-microbial peptide LL-37 is involved in re- 
epithelialization of human skin wounds and is lacking in chronic ulcer epithelium. J. Invest. Dermatol. 120, 379-389

9 Koczulla, R., von Degenfeld, G., Kupatt, C., Krotz, F., Zahler, S., Gloe, T., Issbrucker, K., Unterberger, P., Zaiou, M., Lebherz, C., Karl, A., Raake, P., Pfosser, A., Boekstegers, P., Welsch, U., Hiemstra, P. S., Vogelmeier, C., Gallo, R. L., Clauss, M. and Bals, R. (2003) An angiogenic role for the human peptide antibiotic LL-37/hCAP-18. J. Clin. Invest. 111, 1665-1672

10 Tomasinsig, L., Pizzirani, C., Skerlavaj, B., Pellegatti, P., Gulinelli, S., Tossi, A., Di Virgilio, F. and Zanetti, M. (2008) The human cathelicidin LL-37 modulates the activities of the P2X7 receptor in a structure-dependent manner. J. Biol. Chem. 283, 30471-30481

11 Wang, G. (2008) Structures of human host defense cathelicidin LL-37 and its smallest antimicrobial peptide KR-12 in lipid micelles. J. Biol. Chem. 283, 32637-32643

12 Johansson, J., Gudmundsson, G. H., Rottenberg, M. E., Berndt, K. D. and Agerberth, B. (1998) Conformation-dependent antibacterial activity of the naturally occurring human peptide LL37. J. Biol. Chem. 273, 3718-3724

13 Tomasinsig, L., Morgera, F., Antcheva, N., Pacor, S., Skerlavaj, B., Zanetti, M. and Tossi, A. (2009) Structure dependence of biological activities for primate cathelicidins. J. Pept. Sci. 15, 576-582

14 Zhao, C., Nguyen, T., Boo, L. M., Hong, T., Espiritu, C., Orlov, D., Wang, W., Waring, A. and Lehrer, R. I. (2001) RL-37, an alpha-helical antimicrobial peptide of the rhesus monkey. Antimicrob. Agents Chemother. 45, 2695-2702

15 Morgera, F., Vaccari, L., Antcheva, N., Scaini, D., Pacor, S. and Tossi, A. (2009) Primate cathelicidin orthologues display different structures and membrane interactions. Biochem. J. 417, 727-735

16 Pag, U., Oedenkoven, M., Sass, V., Shai, Y., Shamova, O., Antcheva, N., Tossi, A. and Sahl, H. G. (2008) Analysis of in vitro activities and modes of action of synthetic antimicrobial eptides derived from an alpha-helical 'sequence template'. J. Antimicrob. Chemother. 61, 341-352

17 Tucker, M. J., Oyola, R. and Gai, F. (2006) A novel fluorescent probe for protein binding and folding studies: p-cyano-phenylalanine. Biopolymers. 83, 571-576

18 Aprilakis, K. N., Taskent, H. and Raleigh, D. P. (2007) Use of the novel fluorescent amino acid p-cyanophenylalanine offers a direct probe of hydrophobic core formation during the folding of the N-terminal domain of the ribosomal protein L9 and provides evidence for two-state folding. Biochemistry. 46, 12308-12313

19 Miyake-Stoner, S. J., Miller, A. M., Hammill, J. T., Peeler, J. C., Hess, K. R., Mehl, R. A. and Brewer, S. H. (2009) Probing protein folding using site-specifically encoded unnatural amino acids as FRET donors with tryptophan. Biochemistry. 48, 5953-5962

20 Marek, P., Gupta, R. and Raleigh, D. P. (2008) The fluorescent amino acid pcyanophenylalanine provides an intrinsic probe of amyloid formation. Chembiochem. 9, 1372-1374

21 Tucker, M. J., Tang, J. and Gai, F. (2006) Probing the kinetics of membrane-mediated helix folding. J. Phys. Chem. B. 110, 8105-8109

22 Pazos, I. M., Roesch, R. M. and Gai, F. (2013) Quenching of -Cyanophenylalanine Fluorescence by Various Anions. Chem. Phys. Lett. 563, 93-96

23 Taskent-Sezgin, H., Marek, P., Thomas, R., Goldberg, D., Chung, J., Carrico, I. and Raleigh, D. P. (2010) Modulation of p-cyanophenylalanine fluorescence by amino acid side chains and rational design of fluorescence probes of alpha-helix formation. Biochemistry. 49, 6290-6295

24 Serrano, A. L., Bilsel, O. and Gai, F. (2012) Native state conformational heterogeneity of HP35 revealed by time-resolved FRET. J. Phys. Chem. B. 116, 10631-10638

25 Serrano, A. L., Troxler, T., Tucker, M. J. and Gai, F. (2010) Photophysics of a Fluorescent Non-natural Amino Acid: p-Cyanophenylalanine. Chem. Phys. Lett. 487, 303-306

26 Tucker, M. J., Getahun, Z., Nanda, V., DeGrado, W. F. and Gai, F. (2004) A new method for determining the local environment and orientation of individual side chains of membranebinding peptides. J. Am. Chem. Soc. 126, 5078-5079 
27 Chen, Y. H., Yang, J. T. and Chau, K. H. (1974) Determination of the helix and beta form of proteins in aqueous solution by circular dichroism. Biochemistry. 13, 3350-3359

28 Morgera, F., Antcheva, N., Pacor, S., Quaroni, L., Berti, F., Vaccari, L. and Tossi, A. (2008) Structuring and interactions of human beta-defensins 2 and 3 with model membranes. J. Pept. Sci. 14, 518-523

29 Silvestro, L. and Axelsen, P. H. (1998) Infrared spectroscopy of supported lipid monolayer, bilayer, and multibilayer membranes. Chem. Phys. Lipids. 96, 69-80

30 Besenicar, M., Macek, P., Lakey, J. H. and Anderluh, G. (2006) Surface plasmon resonance in protein-membrane interactions. Chem. Phys. Lipids. 141, 169-178

31 Anderluh, G., Besenicar, M., Kladnik, A., Lakey, J. H. and Macek, P. (2005) Properties of nonfused liposomes immobilized on an L1 Biacore chip and their permeabilization by a eukaryotic pore-forming toxin. Anal. Biochem. 344, 43-52

32 Zelezetsky, I., Pacor, S., Pag, U., Papo, N., Shai, Y., Sahl, H. G. and Tossi, A. (2005) Controlled alteration of the shape and conformational stability of alpha-helical cell-lytic peptides: effect on mode of action and cell specificity. Biochem. J. 390, 177-188

33 Stryer, L. and Haugland, R. P. (1967) Energy transfer: a spectroscopic ruler. Proc. Natl. Acad. Sci. U.S.A. 58, 719-726

34 Tucker, M. J., Oyola, R. and Gai, F. (2005) Conformational distribution of a 14-residue peptide in solution: a fluorescence resonance energy transfer study. J. Phys. Chem. B. 109, 47884795

35 Lakowicz, J. R. (2006) Principles of fluorescence specrtoscopy.

36 Blanco-Rodriguez, A. M., Towrie, M., Sykora, J., Zalis, S. and Vlcek, A., Jr. (2011) Photoinduced intramolecular tryptophan oxidation and excited-state behavior of $[\operatorname{Re}(\mathrm{L}-$ $\mathrm{AA})(\mathrm{CO}) 3$ (alpha-diimine $)](+)(\mathrm{L}=$ pyridine or imidazole, $\mathrm{AA}=$ tryptophan, tyrosine, phenylalanine). Inorg. Chem. 50, 6122-6134

37 Oren, Z., Lerman, J. C., Gudmundsson, G. H., Agerberth, B. and Shai, Y. (1999) Structure and organization of the human antimicrobial peptide LL-37 in phospholipid membranes: relevance to the molecular basis for its non-cell-selective activity. Biochem J. 341 ( Pt 3), 501-513

38 Zelezetsky, I., Pontillo, A., Puzzi, L., Antcheva, N., Segat, L., Pacor, S., Crovella, S. and Tossi, A. (2006) Evolution of the primate cathelicidin. Correlation between structural variations and antimicrobial activity. J. Biol. Chem. 281, 19861-19871

39 Wagschal, K., Tripet, B., Lavigne, P., Mant, C. and Hodges, R. S. (1999) The role of position a in determining the stability and oligomerization state of alpha-helical coiled coils: 20 amino acid stability coefficients in the hydrophobic core of proteins. Prot. Sci. 8, 2312-2329

40 Mukherjee, S., Chowdhury, P., DeGrado, W. F. and Gai, F. (2007) Site-specific hydration status of an amphipathic peptide in AOT reverse micelles. Langmuir. 23, 11174-11179

41 Shai, Y. (1999) Mechanism of the binding, insertion and destabilization of phospholipid bilayer membranes by alpha-helical antimicrobial and cell non-selective membrane-lytic peptides. Biochim. Biophys. Acta. 1462, 55-70

42 Vandamme, D., Landuyt, B., Luyten, W. and Schoofs, L. (2012) A comprehensive summary of LL-37, the factotum human cathelicidin peptide. Cell. Immunol. 280, 22-35 